\title{
A RIEMANN-ROCH THEOREM FOR EDGE-WEIGHTED GRAPHS
}

\author{
RODNEY JAMES AND RICK MIRANDA \\ (Communicated by Ted Chinburg)
}

\begin{abstract}
We prove a Riemann-Roch theorem for real divisors on edgeweighted graphs over the reals, extending the result of Baker and Norine for integral divisors on graphs with multiple edges.
\end{abstract}

\section{INTRODUCTION}

The purpose of this article is to prove a Riemann-Roch theorem for edge-weighted graphs, inspired by (and extending) the theorem of Baker and Norine (see [1]). In that context, graphs without loops but with multiple edges are considered. We consider the existence of multiple edges to be equivalent to assigning to each pair of vertices an integral weight which records the number of edges between them. In our setting we consider arbitrary positive real numbers as edge weights. This variation forces several interesting adjustments to be made to the theory.

Let $R$ be a subring of the real numbers $\mathbb{R}$. An $R$-graph $G$ is a finite connected graph (without loops or multiple edges) where each edge is assigned a weight, which is a positive element of $R$. If we let the $n$ vertices of $G$ be $\left\{v_{1}, \ldots, v_{n}\right\}$, we will denote by $p_{i j}=p_{j i}$ the weight of the edge joining $v_{i}$ and $v_{j}$. If there is no edge connecting $v_{i}$ and $v_{j}$, we set $p_{i j}=p_{j i}=0$.

We define the degree of a vertex $v_{j}$ of $G$ to be the sum of the weights of the edges incident to it:

$$
\operatorname{deg}\left(v_{j}\right)=\sum_{i \neq j} p_{i j}
$$

The edge-weighted Laplacian matrix $P$ of $G$ is the symmetric $n \times n$ matrix defined by

$$
(P)_{i j}= \begin{cases}-p_{i j} & \text { if } i \neq j, \\ \operatorname{deg}\left(v_{j}\right) & \text { if } i=j .\end{cases}
$$

Note that if each $p_{i j} \in\{0,1\}, P$ is the Laplacian matrix of a regular graph; as is the case for the regular graph Laplacian, $P$ is semi-positive definite with kernel generated by $(1,1, \ldots, 1)$.

The genus of $G$ is defined as

$$
g=\sum_{i<j} p_{i j}-n+1,
$$

which allows $g$ to be negative when the $p_{i j}$ are sufficiently small.

Received by the editors August 4, 2009 and, in revised form, March 30, 2011 and January 27, 2012 .

2010 Mathematics Subject Classification. Primary 05C38, 05C25; Secondary 14C40. 
An $R$-divisor $D$ on $G$ is a formal sum

$$
D=\sum_{i=1}^{n} d_{i} \cdot v_{i}
$$

where each $d_{i} \in R$; the divisors form a free $R$-module $\operatorname{Div}(G)$ of rank $n$. We write $D_{1} \geq D_{2}$ if the inequality holds at each vertex; for a constant $c$, we write $D \geq c$ (respectively $D>c$ ) if $d_{i} \geq c$ (respectively $d_{i}>c$ ) for each $i$.

The degree of a divisor $D$ is

$$
\operatorname{deg}(D)=\sum_{i=1}^{n} d_{i}
$$

and the ceiling of $D$ is the divisor

$$
\lceil D\rceil=\sum_{i=1}^{n}\left\lceil d_{i}\right\rceil \cdot v_{i} .
$$

The degree map is a homomorphism from $\operatorname{Div}(G)$ to $R$, and the kernel $\operatorname{Div}_{0}(G)$ of divisors of degree zero is a free $R$-module of rank $n-1$.

Let $H_{j}=\operatorname{deg}\left(v_{j}\right) \cdot v_{j}-\sum_{i \neq j} p_{i j} \cdot v_{i}$, and set $\operatorname{PDiv}(G)=\left\{\sum_{i=1}^{n} c_{i} H_{i} \mid c_{i} \in \mathbb{Z}\right\}$ to be the free $\mathbb{Z}$-module generated by the $H_{j}$. (Note that the $H_{j}$ divisors correspond to the columns of the matrix $P$.) If $G$ is connected, $\operatorname{PDiv}(G)$ has rank $n-1$.

For two divisors $D, D^{\prime} \in \operatorname{Div}(G)$, we say that $D$ is linearly equivalent to $D^{\prime}$ and write $D \sim D^{\prime}$ if and only if $D-D^{\prime} \in \operatorname{PDiv}(G)$.

The linear system associated with a divisor $D$ is

$$
\begin{aligned}
|D| & =\left\{D^{\prime} \in \operatorname{Div}(G) \mid D \sim D^{\prime} \text { with }\left\lceil D^{\prime}\right\rceil \geq 0\right\} \\
& =\left\{D^{\prime} \in \operatorname{Div}(G) \mid D \sim D^{\prime} \text { with } D^{\prime}>-1\right\} .
\end{aligned}
$$

We note that linearly equivalent divisors have the same linear system. The use of the ceiling divisor in the definition above is the critical difference between this theory and the integral theory developed by Baker and Norine [1]. The following lemma gives a condition for $|D|=\emptyset$.

Lemma 1.1. If $D \leq-1$, then $|D|=\emptyset$.

Proof. Suppose that $D \leq-1$ and thus $\operatorname{deg}(D) \leq-n$. If $|D| \neq \emptyset$, there is an $H \in$ $\operatorname{PDiv}(G)$ such that $H+D>-1$, and thus $\operatorname{deg}(H+D)>-n$. Since $\operatorname{deg}(H)=0$, $\operatorname{deg}(H+D)=\operatorname{deg}(H)+\operatorname{deg}(D)=\operatorname{deg}(D)$; hence we must have $|D|=\emptyset$.

The essence of the Riemann-Roch theorem, for divisors on algebraic curves, is to notice that the linear system corresponds to a vector space of rational functions and to relate the dimensions of two such vector spaces. In our context we do not have vector spaces, so we measure the size of the linear system in a different way (as do Baker and Norine).

Define the $h^{0}$ of an $R$-divisor $D=\sum_{i=1}^{n} d_{i} \cdot v_{i}$ :

$$
h^{0}(D)=\min \{\operatorname{deg}(E) \mid E \text { is an } R \text {-divisor, } E \geq 0 \text { and }|D-E|=\emptyset\} .
$$

Note that $h^{0}(D) \geq 0$, with equality if and only if $|D|=\emptyset$ (since $E \geq 0, \operatorname{deg}(E)=0$ if and only if $E=0$, and thus $|D|=\emptyset)$. We can find an upper bound for $h^{0}(D)$ as follows: set $E=\sum_{i=1}^{n} \max \left\{d_{i}+1,0\right\} \cdot v_{i}$; then $D-E \leq-1$, and by Lemma 1.1 . $|D-E|=\emptyset$ and thus $h^{0}(D) \leq \sum_{i=1}^{n} \max \left\{d_{i}+1,0\right\}$. Since $h^{0}$ is defined to be the 
minimum degree of an $R$-divisor, $h^{0} \in R$; however, we will show that $h^{0}(D)$ does not depend on the change of $R$.

The canonical divisor of $G$ is defined as

$$
K=\sum\left(\operatorname{deg}\left(v_{i}\right)-2\right) \cdot v_{i}
$$

Note that $\operatorname{deg}(K)=2 g-2$.

The Riemann-Roch result that we will prove can now be stated.

Theorem 1.2. Let $G$ be a connected $R$-graph as above, and let $D$ be an $R$-divisor on $G$. Then

$$
h^{0}(D)-h^{0}(K-D)=\operatorname{deg}(D)+1-g .
$$

Since $h^{0}(K-D) \geq 0$, the classical Riemann inequality $h^{0}(D) \geq \operatorname{deg}(D)+1-g$ holds. The results of Baker and Norine (see [1]) are exactly that the above theorem holds in the case of the subring $R=\mathbb{Z}$. Our proof depends on the Baker-Norine Theorem in a critical way; it would be interesting to provide an independent proof.

In [3] and [4, a Riemann-Roch theorem is proved for metric graphs with integral divisors; these results differ from the present result in two fundamental ways. First, our edge weights $p_{i j}$ and the coefficients of the divisors are elements of the ring $R$. Second, the genus $g$ is in $R$ for the present result, whereas in [3] and [4, $g$ is a nonnegative integer.

We close this section with an example. Consider the $R$-graph $G$ with two vertices and edge weight $p>0$. For convenience, we will write the divisor $a \cdot v_{1}+b \cdot v_{2}$ as the ordered pair $(a, b)$. The principal divisors are $\operatorname{PDiv}(G)=\{(n p,-n p) \mid n \in \mathbb{Z}\}$ and $K=(p-2, p-2)$, with $g=p-1$. Note that if $p<1$, we have $g<0$.

For $(a, b) \in \operatorname{Div}(G)$, the linear system $|(a, b)|$ can be written as

$$
\begin{aligned}
|(a, b)| & =\{(c, d) \in \operatorname{Div}(G) \mid\lceil(c, d)\rceil \geq 0 \text { and }(c, d) \sim(a, b)\} \\
& =\{(a+n p, b-n p) \mid n \in \mathbb{Z}, a+n p>-1, b-n p>-1\} .
\end{aligned}
$$

In what follows, we will be brief and leave most of the details to the reader to verify. One can check that $|(a, b)| \neq \emptyset$ if and only if $\lceil(1+a) / p\rceil+\lceil(1+b) / p\rceil \geq 2$. The value of $h^{0}((a, b))$ can be computed as follows: let $\phi_{p}(x)=\lfloor(x+1) / p\rfloor$ and

$$
h^{0}((a, b))= \begin{cases}0 & \text { if } \phi_{p}(a)+\phi_{p}(b)<0 \\ \min \left\{a+1-p \phi_{p}(a), b+1-p \phi_{p}(b)\right\} & \text { if } \phi_{p}(a)+\phi_{p}(b)=0 \\ a+b-p+2 & \text { if } \phi_{p}(a)+\phi_{p}(b)>0\end{cases}
$$

Note that for the divisor $D=(0,0)$, we have

$$
h^{0}((0,0))= \begin{cases}2-p & \text { if } p \leq 1 \\ 1 & \text { if } p>1\end{cases}
$$

and that the classical inequality $h^{0}(D) \leq \operatorname{deg}(D)+1$ does not hold when $p<1$.

To check that the Riemann-Roch formula holds for a divisor $D=(a, b)$, it is easiest to consider the three cases for the formula for $h^{0}((a, b))$. We note that $(a, b)$ is in one of the three cases if and only if $(p-2-a, p-2-b)$ is in the opposite case. 
It is very straightforward then to check Riemann-Roch in case $\phi_{p}(a)+\phi_{p}(b) \neq 0$; one of the two $h^{0}$ values is zero. It is a slightly more interesting exercise, but still straightforward, to check it in case $\phi_{p}(a)+\phi_{p}(b)=0$.

Unfortunately, the method of direct computation in this example becomes intractable for $R$-graphs with $n>2$.

\section{Change of Rings}

Note that in the definition of the $h^{0}$ of a divisor, the minimum is taken over all nonnegative $R$-divisors. Therefore, a priori, the definition of $h^{0}$ depends on the subring $R$. We note that if $R \subset S \subset \mathbb{R}$ are two subrings of $\mathbb{R}$, then any $R$-graph $G$ and $R$-divisor $D$ on $G$ are also an $S$-graph and an $S$-divisor. In this section we will see that the $h^{0}$ in fact does not depend on the subring.

Any $H \in \operatorname{PDiv}(G)$ can be written as an integer linear combination of any $n-1$ elements of the set $\left\{H_{1}, H_{2}, \ldots, H_{n}\right\}$. If we exclude $H_{k}$, for example, then there are $n-1$ integers $\left\{m_{j}\right\}_{j \neq k}$ such that $H=\sum_{j \neq k} m_{j} H_{j}$, and we can write $H=$ $\sum_{i=1}^{n} h_{i} \cdot v_{i}$, where

$$
h_{i}=\left\{\begin{array}{cl}
m_{i} \operatorname{deg}\left(v_{i}\right)-\sum_{j \neq k, i} m_{j} p_{i j} & \text { if } i \neq k, \\
-\sum_{j \neq k} m_{j} p_{j k} & \text { if } i=k .
\end{array}\right.
$$

Let $P_{k}$ be the $(n-1) \times(n-1)$ matrix obtained by deleting the $k$ th row and column from the matrix $P$. We can write the $h_{i}$ 's other than $h_{k}$ in matrix form as $\mathbf{h}=P_{k} \mathbf{m}$, where $\mathbf{h}=\left(h_{i}\right)_{i \neq k}$ and $\mathbf{m}=\left(m_{i}\right)_{i \neq k}$ are the corresponding column vectors.

For any $\mathbf{x}=\left(x_{i}\right) \in \mathbb{R}^{n-1}$ and $c \in \mathbb{R}$, we say $\mathbf{x} \geq c$ if and only if $x_{i} \geq c$ for each $i$. Similarly for a matrix $A=\left(a_{i j}\right)$, we write $A \geq c$ if and only if $a_{i j} \geq c$ for each $i, j$.

A $(n-1) \times(n-1)$ matrix $M$ is monotone if $M \mathbf{x} \geq 0$ implies that $\mathbf{x} \geq 0$ for all $\mathbf{x} \in \mathbb{R}^{n-1}$. If $M$ is monotone, it follows that $M$ is nonsingular, with $M^{-1} \geq 0$ (see Chapter 6 in [2]).

Lemma 2.1. $P_{k}$ is monotone.

Proof. Let $V_{i}=\left\{i^{\prime} \mid p_{i i^{\prime}}>0, i^{\prime} \neq k, i^{\prime} \neq i\right\}$ be the set of indices of vertices connected to $v_{i}$ (excluding $k$ ). Suppose that it is the case that $x_{i}<0$ and that $x_{i} \leq x_{i^{\prime}}$ for all $i^{\prime} \in V_{i}$. Then

$$
\begin{aligned}
\left(P_{k} \mathbf{x}\right)_{i} & =x_{i} \operatorname{deg}\left(v_{i}\right)-\sum_{i^{\prime} \in V_{i}} x_{i^{\prime}} p_{i i^{\prime}} \\
& =x_{i} p_{i k}+x_{i} \sum_{i^{\prime} \in V_{i}} p_{i i^{\prime}}-\sum_{i^{\prime} \in V_{i}} x_{i^{\prime}} p_{i i^{\prime}} \\
& =x_{i} p_{i k}+\sum_{i^{\prime} \in V_{i}} p_{i i^{\prime}}\left(x_{i}-x_{i^{\prime}}\right),
\end{aligned}
$$

and we note that with our assumptions, no term here is positive. Since the sum is nonnegative, we conclude that all terms are zero. We have verified the following; therefore, if $P_{k} \mathbf{x} \geq 0$ :

$$
x_{i}<0 \text { and } x_{i} \leq x_{i^{\prime}} \text { for all } i^{\prime} \in V_{i} \Rightarrow p_{i k}=0 \text { and } x_{i}=x_{i^{\prime}} \text { for all } i^{\prime} \in V_{i} .
$$


Now assume that $\mathbf{x} \nsupseteq 0$; then there is an index $j$ such that $x=x_{j}<0$ and $x_{j} \leq x_{i}$ for all $i \neq k$. By (2.2), we conclude that $x_{i}=x$ for all $i \in V_{j}$ and also that $p_{j k}=0$. We see, by induction on the distance in $G$ to the vertex $v_{j}$, that we must have $x_{i}=x$ and $p_{i j}=0$ for all $i \neq k$. This contradicts the connectedness of $G$ : the vertex $v_{k}$ has no edges on it. Thus $P_{k}$ is monotone.

We can now prove the main result for this section.

Proposition 2.2. Suppose that all of the entries of the matrix $P$ are in two subrings $R$ and $R^{\prime}$ and that all the coordinates of the divisor $D$ are also in both $R$ and $R^{\prime}$. Then (using the obvious notation) $h^{0}=h^{0^{\prime}}$.

Proof. It suffices to prove the statement when one of the subrings is $R$ and the other is $\mathbb{R}$. In this case we'll use the notation $R h^{0}$ and $\mathbb{R} h^{0}$, respectively, for the two minima in question.

First note that the linear system $|D|$ is clearly independent of the ring; in particular, whether a linear system is empty or not is also independent.

Therefore, the minimum in question for the $\mathbb{R} h^{0}$ computation is over a strictly larger set of divisors; hence there can only be a smaller minimum. This proves that $R h^{0}(D) \geq \mathbb{R} h^{0}(D)$.

Suppose that $E$ is an $\mathbb{R}$-divisor, $E \geq 0$, and $|D-E|=\emptyset$, achieving the minimum, so that $\mathbb{R} h^{0}(D)=\operatorname{deg}(E)$. If $E$ is an $R$-divisor, it also achieves the minimum in $R$ and $R h^{0}(D)=\mathbb{R} h^{0}(D)$. We will show that in fact $E$ must be an $R$-divisor.

Now suppose that $E$ is not an $R$-divisor, and write $D=\sum_{i=1}^{n} d_{i} \cdot v_{i}$ and $E=$ $\sum_{i=1}^{n} e_{i} \cdot v_{i}$, with $k$ the index of an element such that $e_{k} \notin R$. Since $\mathbb{R} h^{0}(D)=$ $\operatorname{deg}(E)$, for any $\epsilon \in \mathbb{R}$ with $0<\epsilon \leq e_{k}$, we have that $E-\epsilon \cdot v_{k} \geq 0$, and therefore $\left|D-E+\epsilon \cdot v_{k}\right| \neq \emptyset$. Hence there are principal divisors $H$ such that $D-E+\epsilon \cdot v_{k}+H>$ -1 .

Let $\mathcal{H}_{\epsilon}$ be the set of all such $H$; by assumption, this is a nonempty set. Note that if $H \in \mathcal{H}_{\epsilon}$ and $H=\sum_{i=1}^{n} h_{i} \cdot v_{i}$, then $d_{i}-e_{i}+h_{i}>-1$ for each $i \neq k$, and

$$
d_{k}-e_{k}+\epsilon+h_{k}>-1 \text {. }
$$

Also, since $|D-E|=\emptyset$, there is a $k^{\prime}$ such that $d_{k^{\prime}}-e_{k^{\prime}}+h_{k^{\prime}} \leq-1$; combined with the conditions above, the only possibility is $k^{\prime}=k$. Since $d_{k} \in R, h_{k} \in R$ and $e_{k} \notin R$, $d_{k}-e_{k}+h_{k} \neq-1$, and thus $d_{k}-e_{k}+h_{k}<-1$. Hence $-1-\epsilon<d_{k}-e_{k}+h_{k}<-1$.

For any $H \in \mathcal{H}_{\epsilon}$, there are unique integers $m_{i}$ such that $H=\sum_{i \neq k} m_{i} H_{i}$. Let $\mathbf{d}=\left(d_{i}\right)_{i \neq k}, \mathbf{e}=\left(e_{i}\right)_{i \neq k}$, and $\mathbf{m}=\left(m_{i}\right)_{i \neq k}$ be the corresponding column vectors, and define $\mathbf{f}=\left(f_{i}\right)_{i \neq k}=\mathbf{d}-\mathbf{e}+P_{k} \mathbf{m}$. Note that $\mathbf{f}>-1$, and $h_{k}=-\sum_{i \neq k} m_{k} p_{i k}$ by (2.1).

We can write $\mathbf{m}=P_{k}^{-1}(\mathbf{f}-\mathbf{d}+\mathbf{e})$, and by Lemma 2.1. $P_{k}^{-1} \geq 0$. Therefore, since $\mathbf{e} \geq 0$ and $\mathbf{f}>-1$, the $m_{i}$ are bounded from below; set $M \leq m_{i}$ for all $i \neq k$.

We claim that for $H=\sum_{i \neq k} m_{i} H_{i} \in \mathcal{H}_{\epsilon}$, the possible coordinates

$$
h_{k}=-\sum_{i \neq k} m_{k} p_{i k}
$$

form a discrete set. It will suffice to show that for any real $x$, the possible coordinates $h_{k}$ which are at least $-x$ is a finite set. 
To that end, for any $x \in \mathbb{R}$, set $\mathcal{H}_{\epsilon}(x)=\left\{H \in \mathcal{H}_{\epsilon} \mid \sum_{i \neq k} m_{i} p_{i k} \leq x\right\}$; for large enough $x$ this set is nonempty.

Fix $x \in \mathbb{R}$ such that $\mathcal{H}_{\epsilon}(x) \neq \emptyset$ and choose $j \neq k$ such that $p_{j k}>0$. For $H=\sum_{i \neq k} m_{i} H_{i} \in \mathcal{H}_{\epsilon}(x)$ we then have

$$
M \leq m_{j} \leq \frac{x-\sum_{i \neq j, k} m_{i} p_{i k}}{p_{j k}} \leq \frac{x-M \sum_{i \in V_{k}, i \neq j} p_{i k}}{p_{j k}} .
$$

Thus the coefficients $m_{j} \in \mathbb{Z}$ are bounded both below and above and hence can take on only finitely many values. It follows that the set of possible values of $h_{k}=-\sum_{i \neq k} m_{i} p_{i k}$ is also finite, for $H \in \mathcal{H}_{\epsilon}(x)$. As noted above, this implies that these coordinates $h_{k}$, for $H \in \mathcal{H}_{\epsilon}$, form a discrete set. This in turn implies that there is a maximum value $h$ for the possible $h_{k}$, since for all such $h$ we have $d_{k}-e_{k}+h_{k}<-1$.

Note that if $\epsilon<\epsilon^{\prime}$, then $\mathcal{H}_{\epsilon} \subset \mathcal{H}_{\epsilon^{\prime}}$.

We may now shrink $\epsilon$ (if necessary) to achieve $\epsilon<e_{k}-d_{k}-h-1$. This gives a contradition, since now $d_{k}-e_{k}+\epsilon+h_{k} \leq d_{k}-e_{k}+\epsilon+h<-1$ for $H \in \mathcal{H}_{\epsilon}$, violating (2.3). We conclude that $E$ is in fact an $R$-divisor as desired, finishing the proof.

The result above allows us to simply consider the case of $\mathbb{R}$-graphs.

At the other end of the spectrum, the case of $\mathbb{Z}$-graphs is equivalent to the Baker-Norine theory.

The Baker-Norine dimension of a linear system associated with a divisor $D$ on a graph $G$ defined in [1] is equal to

$$
r(D)=\min \left\{\operatorname{deg}(E) \mid E \in \operatorname{Div}(G), E \geq 0 \text { and }|D-E|_{B N}=\emptyset\right\}-1,
$$

where here the linear system associated with a divisor $D$ is

$$
|D|_{B N}=\left\{D^{\prime} \in \operatorname{Div}(G) \mid D^{\prime} \geq 0 \text { and } D \sim D^{\prime}\right\} .
$$

If we are restricted to $\mathbb{Z}$-divisors on $\mathbb{Z}$-graphs, the $h^{0}$ dimension is compatible with the Baker-Norine dimension:

Lemma 2.3. If $G$ is a $\mathbb{Z}$-graph and $D$ a $\mathbb{Z}$-divisor on $G$, then $h^{0}(D)=r(D)+1$.

Proof. Note that $\lceil D\rceil=D$ since each component of $D$ is in $\mathbb{Z}$. This implies that $|D|=|D|_{B N}$, which gives the result.

\section{Reduction to $\mathbb{Q}$-GRAPhS}

Note that the definition of $h^{0}(D)$ depends on the coordinates of $D$ and on the entries of the matrix $P$ which give the edge weights of the graph $G$. Indeed, the set $\mathcal{E}$ of divisors with empty linear systems depends continuously on $P$ as a subset of $\mathbb{R}^{n}$. (If $\mathcal{F}_{0}$ is the set of divisors $D$ with $d_{i}>-1$ for each $i, \mathcal{E}$ is the complement of the union of all the translates of $\mathcal{F}_{0}$ by the columns of $P$.)

Proposition 3.1. Suppose that the Riemann-Roch Theorem 1.2 is true for connected $\mathbb{Q}$-graphs. Then the Riemann-Roch Theorem is true for connected $\mathbb{R}$-graphs. 
Proof. Assume that Theorem 1.2 holds when $G$ is a $\mathbb{Q}$-graph and $D$ is a $\mathbb{Q}$-divisor.

Suppose that $G$ is an $n$-vertex $\mathbb{R}$-graph and $D$ a $\mathbb{R}$-divisor on $G$. Choose any $\epsilon \in \mathbb{R}$ such that $\epsilon>0$. Since $\mathbb{Q}$ is dense in $\mathbb{R}$, we can choose a $\mathbb{Q}$ divisor $D^{\prime}$ such that

$$
0 \leq D^{\prime}\left(v_{i}\right)-D\left(v_{i}\right)<\frac{\epsilon}{n}
$$

for each vertex $v_{i}$ of $G$. Similarly, we can choose nonnegative edge weights $p_{i j}^{\prime} \in \mathbb{Q}$ such that

$$
\left|p_{i j}-p_{i j}^{\prime}\right|<\frac{2 \epsilon}{n(n-1)}
$$

for each $1 \leq i<j \leq n$, which defines a $\mathbb{Q}$-graph $G^{\prime}$. Let $\operatorname{deg}\left(v_{j}^{\prime}\right)=\sum_{i \neq j} p_{i j}^{\prime}$ be the degree of the $j$ th vertex of $G^{\prime}$, and set $K^{\prime}\left(v_{i}\right)=\operatorname{deg}\left(v_{i}^{\prime}\right)-2$ and $g^{\prime}=\sum_{i<j} p_{i j}^{\prime}-n+1$. We then have $0 \leq \operatorname{deg}\left(D^{\prime}\right)-\operatorname{deg}(D)<\epsilon$ and $\left|g^{\prime}-g\right|<\epsilon$.

From the definition of $h^{0}$ it follows that there is a $\mathbb{R}$-divisor $E$ such that $D-E \geq 0$ with $\operatorname{deg}(D-E)=d$ and $|E|=\emptyset$. Since $h^{0}(D)$ varies continuously with the coordinates of $D$, it follows from Proposition 2.2 that $\operatorname{deg}\left(D^{\prime}-E\right)-\operatorname{deg}(D-E)<\epsilon$, $h^{0}\left(D^{\prime}\right)-h^{0}(D)<\epsilon$. Similarly, $\left|h^{0}\left(K^{\prime}-D^{\prime}\right)-h^{0}(K-D)\right|<2 \epsilon$.

Since Theorem 1.2 holds for $D^{\prime}$,

$$
h^{0}\left(D^{\prime}\right)-h^{0}\left(K^{\prime}-D^{\prime}\right)-\operatorname{deg}\left(D^{\prime}\right)-1+g^{\prime}=0,
$$

which implies that

$$
\left|h^{0}(D)-h^{0}(K-D)-\operatorname{deg}(D)-1+g\right|<5 \epsilon .
$$

Since $\epsilon$ was arbitrary, we have

$$
h^{0}(D)-h^{0}(K-D)-\operatorname{deg}(D)-1+g=0 .
$$

\section{SCALING}

Suppose that $G$ is an $R$-graph with edge weights $p_{i j}$. For any $a>0, a \in R \subset \mathbb{R}$, define $a G$ to be the $R$-graph with the same vertices and with edge weights $\left\{a p_{i j}\right\}$. In other words, if $P$ defines $G$, then $a G$ is the $R$-graph defined by the matrix $a P$.

We will use subscripts to denote with which $R$-graph we are computing, e.g., $|D|_{G}, h_{G}^{0}(D)$, etc., if necessary.

For any divisor $D$ on $G$ and $a>0$, define

$$
T_{a}(D)=a D+(a-1) I,
$$

where

$$
I=\sum_{i} 1 \cdot v_{i}
$$

The transformation $T_{a}$ is a homothety by $a$ centered at $-I$.

Lemma 4.1. Let $D$ be an $R$-divisor. If $a, b>0$ with $a, b \in R$, then the following hold:

(1) $T_{b} \circ T_{b}=T_{a b}$

(2) $T_{a}(D+H)=T_{a}(D)+a H$

(3) $\left.\lceil D\rceil \geq 0 \Leftrightarrow\left\lceil T_{a}(D)\right)\right\rceil \geq 0$

(4) $|D|_{G} \neq \emptyset \Leftrightarrow\left|T_{a}(D)\right|_{a G} \neq \emptyset$

(5) $|D-E|_{G} \neq \emptyset \Leftrightarrow\left|T_{a}(D)-a E\right|_{a G} \neq \emptyset$ 
Proof.

(1) Suppose that $D=\sum_{i} d_{i} \cdot v_{i}$. Then:

$$
\begin{aligned}
T_{a}\left(T_{b}(D)\right) & =T_{a}\left(\sum_{i}\left(b d_{i}+b-1\right) \cdot v_{i}\right) \\
& =\sum_{i}\left(a\left(b d_{i}+b-1\right)+a-1\right) \cdot v_{i} \\
& =\sum_{i}\left(a b d_{i}+a b-a+a-1\right) \cdot v_{i} \\
& =\sum_{i}\left(a b d_{i}+a b-1\right) \cdot v_{i} \\
& =T_{a b}(D) .
\end{aligned}
$$

(2) Let $a>0$ and $D, H \in \operatorname{Div}(G)$; then

$$
\begin{aligned}
T_{a}(D+H) & =a(D+H)+(a-1) I \\
& =a D+a H+(a-1) I \\
& =T_{a}(D)+a H .
\end{aligned}
$$

(3) Let $D=\sum_{i} d_{i} \cdot v_{i} \in \operatorname{Div}(G)$ and $a>0$. Since $T_{a}(D)=\sum_{i}\left(a d_{i}+a-1\right) \cdot v_{i}$, we have

$$
\begin{aligned}
\left.\left\lceil T_{a}(D)\right)\right\rceil \geq 0 & \Leftrightarrow a d_{i}+a-1>-1 \text { for each } i \\
& \Leftrightarrow d_{i}>-1 \text { for each } i \\
& \Leftrightarrow\lceil D\rceil \geq 0 .
\end{aligned}
$$

(4) Suppose $|D|_{G} \neq \emptyset$. Then there is an $H \in \operatorname{PDiv}(G)$ such that $\lceil D+H\rceil \geq 0$. Since $T_{a}(D+H)=T_{a}(D)+a H$ and $a H \in \operatorname{PDiv}(a G)$, by part (3) we have $\left\lceil T_{a}(D)+a H\right\rceil \geq 0$ and thus $\left|T_{a}(D)\right|_{a G} \neq \emptyset$. The converse is an identical argument.

(5) Let $D^{\prime}=D-E$. Then from (4), $\left|D^{\prime}\right|_{G} \neq \emptyset \Leftrightarrow\left|T_{a}\left(D^{\prime}\right)\right|_{a G} \neq \emptyset$, where $T_{a}\left(D^{\prime}\right)=T_{a}(D-E)=T_{a}(D)-a E$.

Corollary 4.2. $h_{a G}^{0}\left(T_{a}(D)\right)=a h_{G}^{0}(D)$.

Proof. Since $a>0$, from Lemma 4.1 (5) we have

$$
\begin{aligned}
h_{a G}^{0}\left(T_{a}(D)\right) & =\min _{E^{\prime} \in \operatorname{Div}(a G)}\left\{\operatorname{deg}\left(E^{\prime}\right)\left|E^{\prime} \geq 0,\right| T_{a}(D)-\left.E^{\prime}\right|_{a G}=\emptyset\right\} \\
& =\min _{E \in \operatorname{Div}(G)}\left\{\operatorname{deg}(a E)|a E \geq 0,| T_{a}(D)-\left.a E\right|_{a G}=\emptyset\right\} \\
& =a\left(\min _{E \in \operatorname{Div}(G)}\left\{\operatorname{deg}(E)|E \geq 0,| T_{a}(D)-\left.a E\right|_{a G}=\emptyset\right\}\right) \\
& =a\left(\min _{E \in \operatorname{Div}(G)}\left\{\operatorname{deg}(E)|E \geq 0,| D-\left.E\right|_{G}=\emptyset\right\}\right) \\
& =a h_{G}^{0}(D) .
\end{aligned}
$$

Lemma 4.3. Let $D$ be an $R$-divisor. If $a>0$ with $a \in R$, then the following hold: (1) $K_{a G}=T_{a}\left(K_{G}\right)+(a-1) I$ 
(2) $K_{a G}-T_{a}(D)=T_{a}\left(K_{G}-D\right)$

(3) $\operatorname{deg}\left(T_{a}(D)\right)=a \operatorname{deg}(D)+(a-1)(n)$

(4) $g_{a G}=a g_{G}+(a-1)(n-1)$

Proof.

(1) Since $K_{a G}=\sum_{i}\left(a \operatorname{deg}\left(v_{i}\right)-2\right) \cdot v_{i}$, we have

$$
\begin{aligned}
T_{a}\left(K_{G}\right) & =T_{a}\left(\sum_{i}\left(\operatorname{deg}\left(v_{i}\right)-2\right) \cdot v_{i}\right) \\
& =a \sum_{i}\left(\operatorname{deg}\left(v_{i}\right)-2\right) \cdot v_{i}+\sum_{i}(a-1) \cdot v_{i} \\
& =\sum_{i}\left(a \operatorname{deg}\left(v_{i}\right)-2 a+a-1\right) \cdot v_{i} \\
& =\sum_{i}\left(a \operatorname{deg}\left(v_{i}\right)-a-1\right) \cdot v_{i} \\
& =K_{a G}-(a-1) I .
\end{aligned}
$$

(2)

$$
\begin{aligned}
K_{a G}-T_{a}(D) & =T_{a}\left(K_{G}\right)+(a-1) I-T_{a}(D) \\
& =a K_{G}+(a-1) I+(a-1) I-a D-(a-1) I \\
& =a\left(K_{G}-D\right)+(a-1) I \\
& =T_{a}\left(K_{G}-D\right) .
\end{aligned}
$$

(3)

$$
\begin{aligned}
\operatorname{deg}\left(T_{a}(D)\right) & =\operatorname{deg}(a D+(a-1) I) \\
& =a \operatorname{deg}(D)+(a-1) \operatorname{deg}(I) \\
& =a \operatorname{deg}(D)+(a-1)(n)
\end{aligned}
$$

(4)

$$
\begin{aligned}
g_{a G} & =\sum_{i} a p_{i j}-n+1 \\
& =a \sum_{i} p_{i j}-a n+a+(a-1) n+1-a \\
& =a g_{G}+(a-1)(n-1) .
\end{aligned}
$$

\section{ReduCtion to $\mathbb{Z}$-GRAPHS}

Theorem 5.1. Let $a>0$. Then

$$
h_{G}^{0}(D)-h_{G}^{0}\left(K_{G}-D\right)=\operatorname{deg}(D)-g_{G}+1
$$

if and only if

$$
h_{a G}^{0}\left(T_{a}(D)\right)-h_{a G}^{0}\left(K_{a G}-T_{a}(D)\right)=\operatorname{deg}\left(T_{a}(D)\right)-g_{a G}+1 .
$$

Proof. Let $a>0$. Multiplying (5.1) by $a$, we have

$$
a h_{G}^{0}(D)-a h_{G}^{0}\left(K_{G}-D\right)=a \operatorname{deg}(D)-a g_{G}+a .
$$


The left side of this equation is equal to

$$
h_{a G}^{0}\left(T_{a}(D)\right)-h_{a G}^{0}\left(T_{a}\left(K_{G}-D\right)\right)=h_{a G}^{0}\left(T_{a}(D)\right)-h_{a G}^{0}\left(K_{a G}-T_{a}(D)\right)
$$

using Corollary 4.2 and Lemma 4.3 (2). The right side of the equation is

$$
\operatorname{deg}\left(T_{a}(D)\right)-(a-1)(n)-g_{a G}+(a-1)(n-1)+a=\operatorname{deg}\left(T_{a}(D)\right)-g_{a G}+1
$$

using Lemma 4.3 (3) and (4). This proves that (5.1) implies (5.2); the converse is identical.

Corollary 5.2. Suppose that the Riemann-Roch Theorem 1.2 is true for connected $\mathbb{Z}$-graphs. Then the Riemann-Roch Theorem is true for connected $\mathbb{Q}$-graphs.

Proof. Given a connected $\mathbb{Q}$-graph $G$ and a $\mathbb{Q}$-divisor $D$ on it, there is an integer $a>0$ such that $a G$ is a connected $\mathbb{Z}$-graph and $T_{a}(D)$ is a $\mathbb{Z}$-divisor. Therefore, by hypothesis, the Riemann-Roch statement (5.2) holds. Hence by Theorem 5.1, (5.1) holds, which is the Riemann-Roch theorem for $D$ on $G$.

We now have the ingredients to prove Theorem 1.2

Proof. First, we note again that the Riemann-Roch Theorem of [1] is equivalent to the Riemann-Roch theorem for connected $\mathbb{Z}$-graphs in our terminology. Therefore, using Corollary 5.2, we conclude that the Riemann-Roch Theorem is true for connected $\mathbb{Q}$-graphs. Then, using Proposition 3.1, we conclude that Riemann-Roch holds for connected $\mathbb{R}$-graphs.

Finally, Proposition 2.2 finishes the proof of the Riemann-Roch theorem for divisors on arbitrary $R$-graphs, for any subring $R \subset \mathbb{R}$.

\section{REFERENCES}

[1] Baker, Matthew and Norine, Serguei, Riemann-Roch and Abel-Jacobi Theory on a Finite Graph, Advances in Mathematics 215, 2007, 766-788. MR2355607 (2008m:05167)

[2] Berman, Abraham and Plemmons, Robert J., Nonnegative Matrices in the Mathematical Sciences, SIAM, Philadelphia, PA, 1994. MR1298430 (95e:15013)

[3] Gathmann, Andreas and Kerber, Michael, A Riemann-Roch Theorem in Tropical Geometry, Mathematische Zeitschrift 259, 2008, 217-230. MR2377750 (2009a:14014)

[4] Mikhalkin, Grigory and Zharkov, Ilia, Tropical Curves, Their Jacobians, and Theta Functions, Comtemporary Mathematics 465, 2008, 203-231. MR2457739(2011c:14163) 80523

Department of Mathematics, Colorado State University, Fort Collins, Colorado

Current address: Department of Mathematical and Statistical Sciences, University of Colorado, Denver, Colorado 80217-3364

Department of Mathematics, Colorado State University, Fort Collins, Colorado 80523 